\title{
Electrical Neuroimaging of Voluntary Audiospatial Attention: Evidence for a Supramodal Attention Control Network
}

\author{
Jessica J. Green, ${ }^{1}$ Sam M. Doesburg, ${ }^{2,3}$ Lawrence M. Ward, ${ }^{4,5}$ and John J. McDonald ${ }^{1}$ \\ ${ }^{1}$ Department of Psychology, Simon Fraser University, Burnaby, British Columbia, Canada V5A 1S6, ${ }^{2}$ Child and Family Research Institute, Vancouver, \\ British Columbia, Canada V5Z 4H4, and ${ }^{3}$ Department of Pediatrics, ${ }^{4}$ Psychophysics and Cognitive Neuroscience Laboratory, Department of Psychology, \\ and ${ }^{5}$ Brain Research Centre, University of British Columbia, Vancouver, British Columbia, Canada V6T 1Z4
}

Previous attempts to investigate the supramodal nature of attentional control have focused primarily on identifying neuroanatomical overlap in the frontoparietal systems activated during voluntary shifts of spatial attention in different sensory modalities. However, the activation of the same neural structures is insufficient evidence for a supramodal system, as the same brain regions could interact with one another in very different ways during shifts of attention in different modalities. Thus, to explore the similarity of the functional networks, it is necessary to identify the neural structures involved and to examine the timing and sequence of activities within the network. To this end, we used an electrical neuroimaging technique to localize the neural sources of electroencephalographic signals recorded from human subjects during audiospatial shifts of attention and to examine the timing and sequence of activities within several regions of interest. We then compared the results to an analogous study of visuospatial attention shifts. Similar frontal and parietal regions were activated during visual and auditory shifts of attention, and the timing of activities within these regions was nearly identical. Following this modality-independent sequence of attention-control activity, activity in the relevant sensory cortex was enhanced in anticipation of the response-relevant target. These results are consistent with the hypothesis that a single supramodal network of frontal and parietal regions mediates voluntary shifts of spatial attention and controls the flow of sensory information in modality-specific sensory pathways.

\section{Introduction}

Voluntarily shifting attention to a spatial location in anticipation of a stimulus will enhance our ability to detect or discriminate visual stimuli appearing at that location, relative to stimuli appearing at unattended locations (Kastner and Ungerleider, 2000). This enhancement of perception and performance is thought to be the end result of control operations performed by a network of frontal and parietal regions that modulates the transmission of information in sensory-specific pathways. Functional neuroimaging methods, such as fMRI, have been used to identify the cortical regions involved in controlling voluntary shifts of attention in visual space (Corbetta and Shulman, 2002; Corbetta et al., 2008) and auditory space (Wu et al., 2007), and there appears to be some overlap in the neural architecture of the visual and auditory attention-control networks (Salmi et al., 2007). The activation of similar brain structures in auditory and visual tasks might indicate that a single, supramodal attention-control network mediates the transmission of information within each

Received Nov. 2, 2010; revised Dec. 2, 2010; accepted Jan. 4, 2011.

This work was supported by grants from the Natural Sciences and Engineering Research Council of Canada, the Canadian Foundation for Innovation, the British Columbia Knowledge Development Fund, and the Canada Research Chairs program. We thank Viola Störmer for assistance with data collection.

Correspondence should be addressed to Jessica J. Green, Center for Cognitive Neuroscience, Duke University, Box 90999, Durham, NC 27708. E-mail: jessica_green@alumni.sfu.ca.

DOI:10.1523/JNEUROSCI.5758-10.2011

Copyright $\odot 2011$ the authors $\quad 0270-6474 / 11 / 313560-05 \$ 15.00 / 0$ modality-specific sensory pathway (Ward et al., 1998; Wright and Ward, 2008). However, the pictures emerging from the subtractive fMRI method provide little information about the dynamics of the neurophysiological activities associated with cognitive function. Without additional methods to assess spatiotemporal patterns of activations in the networks, it is unknown whether individual regions of the presumed network perform the same neurocognitive operations across two different experimental contexts (McIntosh, 2000). Thus, to determine whether the same functional network is responsible for shifting attention in visual and auditory space, the timing and sequences of the neurocognitive operations associated with attention control must also be examined.

To obtain information about both the neural architecture and the timing of operations that underlie the control and deployment of attention in space, we used an electrical neuroimaging procedure that enabled us to identify active cortical regions from a scalp-recorded electroencephalogram (EEG) (Green and McDonald, 2008, 2009). ${ }^{a}$ In a previous study, we examined the time course of attention control in vision by localizing theta-band (4-7 Hz) EEG activity and then examining the timing and se-

${ }^{a}$ We use the term electrical neuroimaging to connote a spatiotemporal analysis of EEG that takes place primarily in source space rather than sensor space. Other researchers have used the term electrical neuroimaging to refer to different spatiotemporal analyses of EEG activities and their estimated neural sources (Grave de Peralta Menendez et al., 2004). 


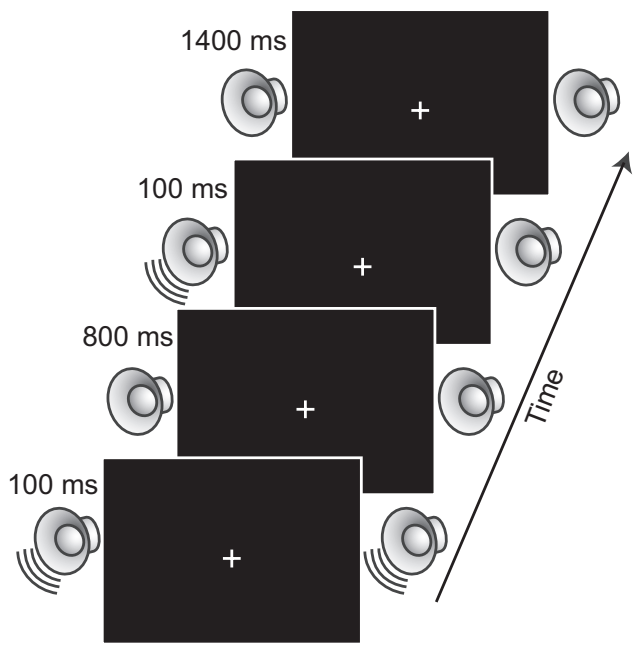

Figure 1. Illustration of events on a single trial.

quence of activities in those regions (Green and McDonald, 2008). An initial feedforward sweep of attention-control activity from occipital regions, through parietal regions, to frontal regions, was followed by a second phase of activity in parietal cortex and a subsequent boost of activity in ventral occipital regions in anticipation of the visual target. In the present study, we examined theta-band EEG activity during shifts of attention within the auditory modality. If audiospatial shifts of attention recruit a supramodal attention-control network, then we would expect to observe the same sequence of parietal and frontal cortical activities as in our previous study of visuospatial attention, but this control activity would lead to anticipatory pretarget biasing in auditory cortex, rather than visual cortex, as participants were required to prepare for task-relevant auditory stimuli.

\section{Materials and Methods}

Participants. Seventeen university students participated in the experiment after providing informed written consent. Data from three participants were not included due to excessive eye movements or blinks. Of the remaining 14 participants (mean age, 20.1 years; SD, \pm 1.9 years; eight females), all were right-handed and reported normal or corrected-to-normal vision and normal hearing. The experimental protocol was approved by the Simon Fraser University ethics committee. An analysis of event-related potentials (ERPs) from these participants was reported previously (Störmer et al., 2009).

Stimuli and apparatus. Participants sat in an acoustically attenuated and electrically shielded chamber. A 19" CRT monitor with a loudspeaker mounted to each side was positioned $65 \mathrm{~cm}$ in front of the participants' head position. Throughout the experimental blocks, a small gray cross $\left(0.9^{\circ} \times 0.9^{\circ}\right)$ was presented in the center of the otherwise unlit CRT display to serve as a fixation point. Auditory stimuli were presented from loudspeakers positioned to the left and right of the monitor. Shift cue stimuli were upward and downward frequency sweeps of sound that ranged from 750 to $1250 \mathrm{~Hz}$. The no-shift cue stimulus consisted of the simultaneous presentation of an upward $(750-1000 \mathrm{~Hz})$ and a downward $(1250-1000 \mathrm{~Hz})$ frequency sweep so that it contained all the same frequencies as the informative cues. Cues were presented from both speakers simultaneously such that they were perceived to be emanating from the center of the computer display. Target sounds were bursts of white noise with either a 5 or $20 \mathrm{~ms}$ gap of silence in the middle. Cue and target stimuli were each $100 \mathrm{~ms}$ in duration, including $5 \mathrm{~ms}$ linear rise and fall times, and $\sim 70 \mathrm{~dB}$ sound pressure level at the location of the participants' ears. A cowbell ( $350 \mathrm{~ms}$ duration, $72 \mathrm{~dB}$ sound pressure level) served as a probe stimulus. All auditory stimuli were digitized at $44 \mathrm{kHz}$.

Procedure. Participants were instructed to maintain their gaze on the fixation cross throughout each experimental block. As shown in Figure 1, each trial began with the $100 \mathrm{~ms}$ presentation of an auditory cue. On shift-cue trials (67\%), participants were instructed to attend to the left or right side depending on the direction of the cue's frequency sweep (50\% left cues, $50 \%$ right cues). For half of the participants, the ascending frequency sweep indicated to attend to the left and the descending frequency sweep indicated to attend to the right, and vice versa for the other half of participants. On no-shift cue trials (33\%), participants were instructed to maintain attention at fixation and not to shift attention to a particular location in anticipation of the target. Following a $900 \mathrm{~ms}$ stimulus-onset asynchrony, an auditory target or probe was presented from either the left or right speaker with equal probability.

The participants' task was to indicate the length of the gap in the target sounds by clicking a mouse button (left, short gap; right, long gap) but to only respond to the probe stimulus when it was presented at the validly cued location (i.e., no response to probes occurring at the uncued location or following a noninformative cue). All participants responded with their right hand. Probe stimuli were presented on one-third of trials. Following the presentation of the target, participants were given a 1400 $\mathrm{ms}$ intertrial interval to respond before the next cue stimulus was presented. The cues were spatially nonpredictive (i.e., 50\% valid) but participants were instructed to attend to the cued location to help them perform the discrimination task. The probe trials were included to ensure that participants were following instructions to attend to the cued location. The experiment consisted of 25 blocks of 36 trials each (24 target trials, 12 probe trials).

Electrophysiological recording. EEG recordings were acquired from 63 tin electrodes using a modified 10-10 system montage (Green et al., 2008) referenced to the right mastoid. Eye movements were monitored using bipolar recordings from electrodes positioned lateral to the left and right external canthi (horizontal electrooculogram). Blinks were detected using scalp electrode FP1, positioned over the left eye. All electrode impedances were kept $<10 \mathrm{k} \Omega$, and signals were recorded with a bandpass of $0.1-100 \mathrm{~Hz}$ ( $-3 \mathrm{~dB}$ point; $-12 \mathrm{~dB}$ per octave) and digitized at 500 $\mathrm{Hz}$. Artifact rejection was performed using a semiautomated procedure (Green et al., 2008) to remove epochs that contained eye movements, blinks, or amplifier blocking. Artifact-free data epochs were then used for further analysis. All participants had a minimum of $70 \%$ of trials retained in each condition after artifact removal.

Beamformer source localization. Beamformer analysis was performed on each participant's data using the Multiple Source Beamformer implemented in the Brain Electrical Source Analysis software (BESA 5.1; Megis Software). This analysis produces a spatially filtered estimate of activity throughout the brain by dividing the brain into a three-dimensional grid and, for each point in the brain, estimating the amount of activity a source at that location would contribute to the activity at the scalp (Van Veen et al., 1997; Gross et al., 2001; Green and McDonald, 2009).

The BESA-implemented beamformer estimates activity specifically in the time-frequency domain, requiring each participant's EEG to first be transformed into a time-frequency signal. The time-frequency transformation was accomplished using the complex demodulation technique implemented in BESA (for more details, see Hoechstetter et al., 2004; Green and McDonald, 2008). Our time-frequency sampling ( $1 \mathrm{~Hz} / 50 \mathrm{~ms})$ results in the signal being smoothed to a time-frequency signal with a full power width at half maximum of frequency $\pm 1.42 \mathrm{~Hz}$ and time $\pm 78.8 \mathrm{~ms}$. Because we did not subtract out the evoked responses in the EEG during transformation to the time-frequency domain, it is possible that some of the activity in lower frequency bands also incorporates evoked responses that encompass a wider frequency range.

Using a standard realistic head model, the beamformer source estimations for each shift condition (leftward or rightward directing cues), normalized relative to activity in the neutral (no-shift) condition, were output for each $2 \times 2 \times 2 \mathrm{~mm}$ voxel of the brain. The beamformer was applied to 18 separate $50 \mathrm{~ms}$ time windows that spanned the cue-target interval for the theta frequency band $(4-7 \mathrm{~Hz})$. For each participant, the beamformer output for the shift-left and shift-right conditions were collapsed by flipping the shift-right data along the $y$-axis (left-right) and averaging it with the shift-left data. This resulted in a single image for each participant with the activity ipsilateral to the cued location over the 


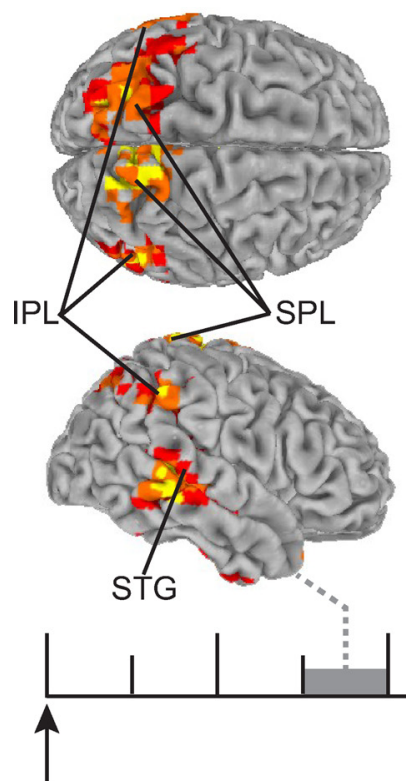

Cue Onset
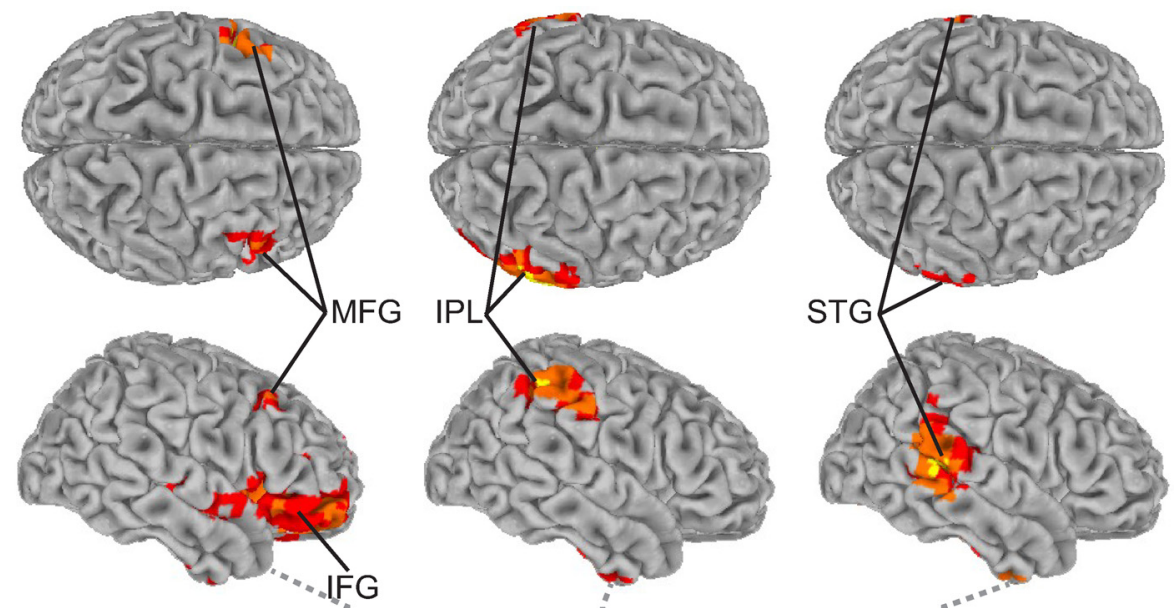

STG

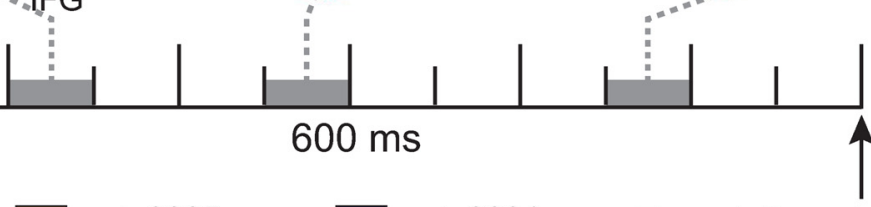

$p<.0005$

$p<.0001$

Target Onset

Figure 2. Spatiotemporal pattern of theta-band (4-7 Hz) activity associated with top-down attention control. Shown are surface-rendered maps of statistically significant increases in activity for shift cues relative to the no-shift cue during four $50 \mathrm{~ms}$ time windows. Activity for shift-left and shift-right cues was collapsed such that the left hemisphere displays activity ipsilateral to the cued location and the right hemisphere displays activity contralateral to the cued location. MFG, middle frontal gyrus.

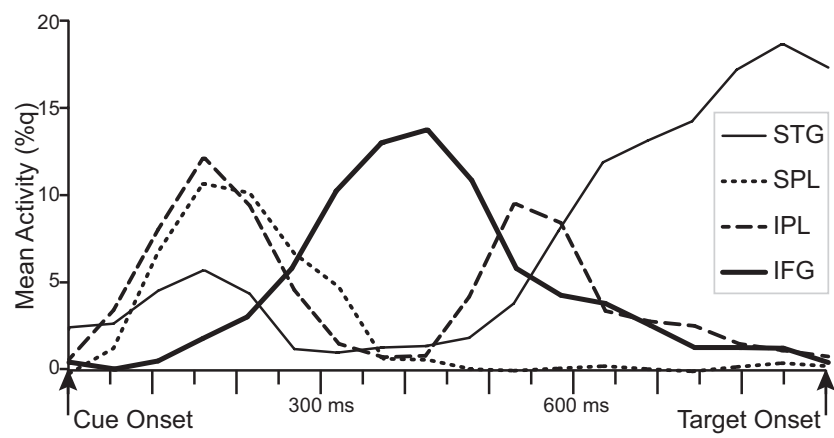

Figure 3. Time courses of beamformer power estimates in auditory, parietal, and frontal ROls. Beamformer time courses are provided in $q$ values, which represent the percentage change in shift-cue activity relative to the neutral-cue activity in the same time interval.

left hemisphere and the activity contralateral to the cued location over the right hemisphere.

For each time interval, the collapsed beamformer output was subjected to a nonparametric statistical analysis using random permutation tests to determine activity significant across participants (Singh et al., $2003)$. The statistically significant activity $(p<0.001)$ was then displayed on a surface-rendered brain using fMRI analysis software (AFNI) (Cox and Hyde, 1997). Regions of interest (ROIs) of $2 \times 2 \times 2 \mathrm{~cm}$ were defined around the centroids of the beamformer source activities to determine, in a data-driven manner, the time courses of attention-control activities within multiple discrete cortical areas. We then assessed the lateralization of effects for each ROI pair [e.g., contralateral-superior parietal lobe (SPL) vs ipsilateral SPL] for each $50 \mathrm{~ms}$ time window. No significant lateralizations of activity were observed for any of the ROI pairs (all $p s>$ 0.09). Because the locations and temporal patterns of activities were nearly identical across hemispheres, we averaged the time courses from the ROIs ipsilateral and contralateral to the attended location together to examine the timing of the bilateral attention control network. Talairach coordinates $(x, y, z)$ of the ROIs were as follows: superior temporal gyrus (STG): $\pm 55,-25,12$; SPL: $\pm 25,-55,61$; inferior parietal lobe (IPL): $\pm 48,-55,34$; and inferior frontal gyrus (IFG): $\pm 48,38,10$. ROI analyses were performed using the raw averaged data, such that output values were the power estimates for the shift-cue condition normalized by the power estimate for the neutral-cue condition in the same time window (average $q$ values) averaged across participants for that ROI.

\section{Results}

Participants were faster to respond to validly cued targets (694 $\mathrm{ms})$ than to invalidly $(716 \mathrm{~ms})$ and neutrally $(718 \mathrm{~ms})$ cued targets and performed satisfactorily on the probe task (hit rate on valid trials, $76 \%$ ), indicating that they did shift attention to the cued location in preparation for the target. No difference in response accuracy was observed (valid, 90.9\%; invalid, 90.6\%; neutral, $90.7 \%$ ), confirming that the response time facilitation on valid-cue trials was not due to a speed-accuracy trade-off (for a full analysis of behavioral results, see Störmer et al., 2009). As can be seen in Figure 2, electrical neuroimaging of theta-band activity revealed a sequential activation of cortical regions during the cue-target interval. Between 150 and 200 ms following the appearance of an attention-directing cue, significant activity was observed in superior temporal, inferior parietal, and superior parietal regions of cortex. Between 350 and 450 ms postcue, activity was observed exclusively in frontal cortex, primarily in inferior frontal regions but also a in a small region of the middle frontal gyrus. Between 500 and 600 ms, activity in frontal cortex dissipated and activity was once again observed in inferior parietal regions. By $700 \mathrm{~ms}$, following this second peak of activity in inferior parietal cortex, activity was observed in superior temporal regions of cortex, which continued until target onset.

To better characterize the spatiotemporal sequences of the beamformer-filtered attention control activities, we plotted the normalized power changes in the beamformer outputs for the shift cues relative to the no-shift cue across the entire cue-target interval in temporal, parietal, and frontal ROIs (Fig. 3 ). Activity in the STG occurred in two phases, with an early peak at $\sim 150-200 \mathrm{~ms}$ after the cue and a late phase that began $\sim 550$ $\mathrm{ms}$ after the cue and continued until the onset of the target stimulus. Activity in the IPL showed a similar biphasic pattern, with 
a

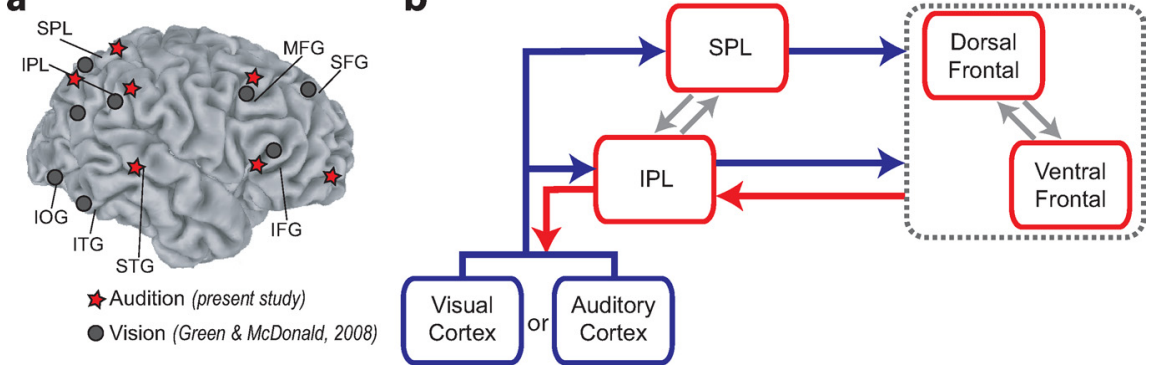

Figure 4. Attention control activities in auditory and visual space. $\boldsymbol{a}, 0$ verview of the neural sources of attention-control-related theta-band EEG activity during shifts of attention in auditory space (present study) and visual space (Green and McDonald, 2008). $\boldsymbol{b}$, Proposed model of supramodal attention control. MFG, Middle frontal gyrus; IOG, inferior occipital gyrus; ITG, inferior temporal gyrus; SFG, superior frontal gyrus.

an early peak $\sim 150 \mathrm{~ms}$ and a late peak around $550 \mathrm{~ms}$. Activity in the SPL peaked early, around the same time as the initial peak activation in IPL, but with no second peak later in the cue-target interval. Activity in the IFG peaked in the middle of the cuetarget interval ( $\sim 300-500 \mathrm{~ms}$ postcue onset) and was minimal during the early and late phases of the cue-target interval. Thus, activities in distinct cortical regions occurred in a specific sequence following the appearance of an attention-directing auditory cue.

\section{Discussion}

The present study used EEG source imaging methods to shed light on the cortical network involved in the control of audiospatial attention. To isolate attention-control activity, theta-band EEG oscillations elicited by spatially uninformative no-shift cues were subtracted from theta-band EEG oscillations elicited by spatially informative shift cues that specified whether participants should covertly direct their attention to the left or right side of fixation in anticipation of an impending auditory target. We then used beamformer spatial filters to locate neural sources of this isolated attention-control activity in successive $50 \mathrm{~ms}$ time intervals following the cue.

This electrical-neuroimaging approach enabled us to make three key observations. First, the deployment of attention in auditory space led to increased theta-band activity in frontal and parietal cortical regions that have been linked to control of visuospatial attention (Hopfinger et al., 2000; Corbetta and Shulman, 2002; Green and McDonald, 2008), providing new evidence that the control activities in these regions are modality independent. Second, the deployment of attention also led to increased thetaband activity in the superior temporal lobes immediately before the appearance of the auditory target. This area of activation included primary and secondary auditory cortices (Brodmann areas 41 and 42) and extended into middle temporal cortices in both hemispheres. Combined with consistent results from recent ERP (Störmer et al., 2009) and neuroimaging (Wu et al., 2007) studies, this result shows that shifting attention to a spatial location in preparation for an expected auditory target leads to anticipatory pretarget biasing within auditory cortices. Third, the timing and sequence of attention-control activity in frontal and parietal regions were nearly identical to those found in a recent EEG study of visuospatial attention using similar methods (Green and McDonald, 2008). In both cases, frontal lobe activity was preceded by activities in cue-specific sensory regions as well as multiple parietal lobe regions. This sequence demonstrates that top-down attention control involves an initial feedforward sweep of information through the attention-control network.
Figure $4 a$ illustrates the regions within which attention-related activities were observed before the appearance of auditory targets (present study) and visual targets (Green and McDonald, 2008). Regardless of sensory modality, attentioncontrol activities were observed in both inferior and superior regions of the parietal and frontal cortices. Previous PET and fMRI studies have implicated these frontoparietal regions in the voluntary control of attention in vision (Corbetta et al., 2000; Corbetta and Shulman, 2002; Macaluso et al., 2003; Serences and Yantis, 2006), audition (Shomstein and Yantis, 2006; Wu et al., 2007; Salmi et al., 2009), and touch (Macaluso et al., 2002, 2003), and in shifting attention between the visual and auditory modalities (modality selection) (Shomstein and Yantis, 2004). The similarities across modalities and tasks suggest that these frontal and parietal regions may perform general attention operations that are modality independent. Such operations would likely involve multimodal neurons that are capable of receiving information from and sending information to multiple sensory modalities. Indeed, multisensory neurons have been found in several cortical regions, and there appears to be considerable overlap between these multisensory brain regions and the voluntary attention control networks (for review, see Macaluso and Driver, 2005).

The observation of attention-control activity in ventral frontoparietal regions is at odds with some existing neuroimaging data. For example, Corbetta and colleagues (2000) reported that activity in more dorsal areas of the posterior parietal cortex (the inferior parietal lobule) was related to voluntary attentional preparation whereas activity in a more ventral parietal area (the temporalparietal junction) was seen only in response to the target stimuli and was greater when the target appeared at an unattended location. Based on these results, Corbetta and Shulman (2002) distinguished between a specialized ventral network for bottom-up attention capture and a specialized dorsal network for top-down control and deployment of attention. However, there is a growing body of evidence linking the ventral frontoparietal regions to top-down control of attention in audition (Alho et al., 1999; Salmi et al., 2009) as well as in vision (Rosen et al., 1999; Peelen et al., 2004). The present results therefore provide converging evidence that the ventral frontoparietal regions are not dedicated to bottom-up attention capture but can also perform top-down attention control operations.

Together with our recent electrical-neuroimaging study of visuospatial attention control (Green and McDonald, 2008), the present results suggest that the following sequence of processing stages occurs during voluntary shifts of spatial attention: (1) attention-related processing of the cue leads to enhanced activity in low-level sensory areas; (2) attention-control activities in inferior and superior parietal areas demarcate the to-be-attended location, provide a signal to switch attention, or both; (3) frontal cortices then initiate executive commands to shift attention to the cued location based on information supplied by the parietal regions; (4) inferior parietal cortex then shifts attention to the cued location and maintains attention there; (5) maintaining attention at the cued location enhances activity in putatively modalityspecific sensory cortices in preparation for the expected target. Processing stages 2 through 4 appears to be common to shifts of spatial attention in both visual and auditory modalities, and thus they likely reflect the operation of a supramodal frontoparietal 
attention network. Stages 1 and 5 occur in modality-specific cortices, but they appear to represent the same general cognitive operations: the attentional modulation of the sensory cortex relevant to the processing of the cue (stage 1) and target (stage 5).

The present results - and the sequence of hypothetical control operations outlined above-are consistent with the model of voluntary attention control presented in Figure $4 b$. Attentioncontrol operations in the frontoparietal regions are largely bilateral, although in the case of visuospatial orienting, some attention-control activities in these areas are lateralized with respect to the to-be-attended location (Green and McDonald, 2008). Given the overlap of the frontoparietal control regions identified here and in a previous study of visuospatial attention (Fig. 4a), the data suggest that the same regions of frontal and parietal cortex may mediate shifts of attention in auditory and visual modalities. However, given the spatial limitation of the electrical neuroimaging approach used here, the present data do not distinguish between this possibility and the alternative that different subregions of frontoparietal cortex participate in the control of auditory attention and visual attention. Either way, the present data do show the same sequence of control activities within the same general frontoparietal network. This control activity can be initiated by any cue stimulus that provides information about a potential location of the impending target. Ultimately, the frontoparietal control operations lead to an anticipatory modulation of neural activity within the modalityspecific sensory pathways that are most relevant for processing the impending target.

\section{References}

Alho K, Medvedev SV, Pakhomov SV, Roudas MS, Tervaniemi M, Reinikainen K, Zeffiro T, Näätänen R (1999) Selective tuning of the left and right auditory cortices during spatially directed attention. Brain Res Cogn Brain Res 7:335-341.

Corbetta M, Shulman GL (2002) Control of goal-directed and stimulusdriven attention in the brain. Nat Rev Neurosci 3:201-215.

Corbetta M, Kincade JM, Ollinger JM, McAvoy MP, Shulman GL (2000) Voluntary orienting is dissociated from target detection in human posterior parietal cortex. Nat Neurosci 3:292-297.

Corbetta M, Patel G, Shulman GL (2008) The reorienting system of the human brain: from environment to theory of mind. Neuron 58:306-324.

Cox RW, Hyde JS (1997) Software tools for analysis and visualization of fMRI data. NMR Biomed 10:171-178.

Grave de Peralta Menendez R, Murray MM, Michel CM, Martuzzi R, Gonzalez Andino SL (2004) Electrical neuroimaging based on biophysical constraints. Neuroimage 21:527-539.

Green JJ, McDonald JJ (2008) Electrical neuroimaging reveals timing of attentional control activity in human brain. PLoS Biol 6:e81.

Green JJ, McDonald JJ (2009) A practical guide to beamformer source reconstruction for EEG. In: Brain signal analysis: advances in neuroelectric and neuromagnetic methods (Handy TC, ed), pp 79-98. Cambridge, MA: MIT.

Green JJ, Conder J, McDonald JJ (2008) Lateralized frontal activity elicited by attention-directing visual and auditory cues. Psychophysiology 45:579-587.

Gross J, Kujala J, Hamalainen M, Timmermann L, Schnitzler A, Salmelin R (2001) Dynamic imaging of coherent sources: studying neural interactions in the human brain. Proc Natl Acad Sci U S A 98:694-699.

Hoechstetter K, Bornfleth H, Weckesser D, Ille N, Berg P, Scherg M (2004) BESA source coherence: a new method to study cortical oscillatory coupling. Brain Topogr 16:233-238.

Hopfinger JB, Buonocore MH, Mangun GR (2000) The neural mechanisms of top-down attentional control. Nat Neurosci 3:284-291.

Kastner S, Ungerleider LG (2000) Mechanisms of visual attention in the human cortex. Annu Rev Neurosci 23:315-341.

Macaluso E, Driver J (2005) Multisensory spatial interactions: a window onto functional integration in the human brain. Trends Neurosci 28:264-271.

Macaluso E, Frith CD, Driver J (2002) Supramodal effects of covert spatial orienting triggered by visual or tactile events. J Cogn Neurosci 14:389-401.

Macaluso E, Eimer M, Frith CD, Driver J (2003) Preparatory states in crossmodal spatial attention: spatial specificity and possible control mechanisms. Exp Brain Res 149:62-74.

McIntosh AR (2000) Towards a network theory of cognition. Neural Netw 13:861-870.

Peelen MV, Heslenfeld DJ, Theeuwes J (2004) Endogenous and exogenous attention shifts are mediated by the same large-scale network. Neuroimage 22:822-830.

Rosen AC, Rao SM, Caffarra P, Scaglioni A, Bobholz JA, Woodley SJ, Hammeke TA, Cunningham JM, Prieto TE, Binder JR (1999) Neural basis of endogenous and exogenous spatial orienting: a functional MRI study. J Cogn Neurosci 11:135-152.

Salmi J, Rinne T, Degerman A, Salonen O, Alho K (2007) Orienting and maintenance of spatial attention in audition and vision: Multimodal and modality-specific brain activations. Brain Struct Funct 212:181-194.

Salmi J, Rinne T, Koistinen S, Salonen O, Alho K (2009) Brain networks of bottom-up triggered and top-down controlled shifting of auditory attention. Brain Res 1286:155-164.

Serences JT, Yantis S (2006) Selective visual attention and perceptual coherence. Trends Cogn Sci 10:38-45.

Shomstein S, Yantis S (2004) Control of attention shifts between vision and audition in human cortex. J Neurosci 24:10702-10706.

Shomstein S, Yantis S (2006) Parietal cortex mediates voluntary control of spatial and nonspatial auditory attention. J Neurosci 26:435-439.

Singh KD, Barnes GR, Hillebrand A (2003) Group imaging of task-related changes in cortical synchronisation using nonparametric permutation testing. Neuroimage 19:1589-1601.

Störmer VS, Green JJ, McDonald JJ (2009) Tracking the voluntary control of auditory spatial attention with event-related brain potentials. Psychophysiology 46:357-366.

Van Veen BD, van Drongelen W, Yuchtman M, Suzuki A (1997) Localization of brain electrical activity via linearly constrained minimum variance spatial filtering. IEEE Trans Biomed Eng 44:867-880.

Ward LM, McDonald JJ, Golestani N (1998) Cross-modal control of attention shifts. In: Visual attention (Wright RD, ed), pp 232-268. New York: Oxford UP.

Wright RD, Ward LM (2008) Crossmodal attention shifts. In: Orienting of attention, pp 199-228. New York: Oxford UO.

Wu CT, Weissman DH, Roberts KC, Woldorff MG (2007) The neural circuitry underlying the executive control of auditory spatial attention. Brain Res 1134:187-198. 